\title{
Posibilidad de uso del Alpechín en Fertilización de Tierras Agrícolas
}

\author{
María T. Pozzi ${ }^{(1)}$; Ana J., Filippín ${ }^{(1)}$; César Matías ${ }^{(2)}$ y Ariadna Hammann ${ }^{(1)}$ \\ (1) Facultad de Ciencias Exactas y Naturales - Universidad Nacional de Catamarca, \\ Av. Belgrano 300. CP 4700 San Fernando del Valle de Catamarca. Catamarca-Argentina \\ (e-mail: mtpozzi02@yahoo.com.ar) \\ (2) EEA.INTA. Ruta Provincial No 33 Km 4. Sumalao. Dpto. Valle Viejo CP 4705.Catamarca- \\ Argentina
}

Recibido Jul. 29, 2009; Aceptado Sept. 04, 2009; Versión Final recibida Nov. 102009

\begin{abstract}
Resumen
En este trabajo se determinan las características del alpechín vertido en una poza y sus modificaciones a través del tiempo para evaluar su posible interacción con el suelo. Se caracterizó el suelo original $\left(S_{0}\right)$, cercano a la poza $\left(S_{1}\right)$ y el alpechín $\left(A_{0}\right)$. Se analizó las modificaciones del efluente $A_{0}$ (el alpechín) a medida que se seca a diferentes tiempos $\left(A_{0}: 0, A_{1}: 10, A_{2}: 100, A_{3}: 190, A_{4}\right.$ : 280 días), transformándose en lodo. Los parámetros se determinaron por triplicado para comparar las diferencias entre alpechín, lodo y suelo. Los parámetros analizados resultaron próximos a los del suelo original, excepto para potasio, materia orgánica y carbono orgánico. Los índices de germinación fueron positivos para dos de las muestras (190 y 280 días). Se infiere que el suelo puede utilizarse como medio para disminuir los efectos del alpechín y se propone a las industrias del sector un método de fertilización sencillo y de bajo costo.
\end{abstract}

Palabras clave: fertilización, alpechín, tierras agrícolas, propiedades del suelo

\section{Possibility of use of the Olive Oil Mill Wastewater in Fertilization of Agricultural Lands}

\begin{abstract}
The characteristics of the disposed olive oil mill wastewater (alpechìn), its possible modifications through time and its interaction with soil are analyzed. The original soil $\left(S_{0}\right)$, the soil near the pond $\left(S_{1}\right)$, and the olive oil mill wastewater $\left(A_{0}\right)$ were characterized. The effluent $A_{0}$ was examined as it dried at different periods $\left(A_{0}: 0, A_{1}: 10, A_{2}: 100, A_{3}: 190, A_{4}: 280\right.$ days). Parameters were determined three times to compare the differences between olive oil mill wastewater, sludge and soil. The parameters proved to be close to those of the original soil except for potassium, organic matter and organic carbon. Index of germination was positive for two of the samples (190 and 280 days). It is concluded that soil can be used as a means for decreasing the olive oil mill wastewater effects proposing to the olive oil industries a simple and low cost fertilization method.
\end{abstract}

Keywords: fertilization, olive mill wastewater, agricultural soil, soil properties 


\section{INTRODUCCIÓN}

En las últimas décadas, la obtención de aceite de oliva se ha convertido en una de las actividades agroindustriales más importantes de la provincia de Catamarca en Argentina. En la campaña 20062007 se produjeron 52.000 toneladas de aceituna; de las cuales aproximadamente el $70 \%$ son destinadas a la elaboración de aceite y el resto para aceituna de mesa. La industria oleícola emplea sistemas de extracción netamente físicos, sin la intervención de sustancias químicas que contaminen los recursos naturales. Sin embargo, genera volúmenes importantes de residuos cuya composición y cantidad dependen del sistema de extracción (Martínez-García et al., 2006); el sistema continuo de tres fases produce por kilogramo de aceituna procesada una cantidad de alpechín que oscila entre 0,5 a 1,4 litros y 0,5 Kg. de orujo (Sierra et al., 2001). En un sistema de dos fases se reduce el consumo de agua y se produce un residuo en forma de pasta (Alpeorujo = alpechín+orujo). La composición del alpechín no es constante tanto cualitativamente como cuantitativamente y varía de acuerdo al suelo cultivado, tiempo de cosecha, grado de maduración del fruto, variedad de aceituna, condiciones climáticas, uso de fertilizantes o de pesticidas (Borja et al., 2006; Niaounakis and Halvadakis, 2004;); y por lo general se caracteriza por ser un líquido de color oscuro, de olor intenso, con alto grado de contaminación orgánica (DQO: Demanda Química de Oxígeno). El pH, levemente ácido, toma valores entre $3-6$; y presenta además, alta conductividad eléctrica (CE), alto contenido de polifenoles (0,5 - $24 \mathrm{~g} / \mathrm{L})$ y alto contenidos de sólidos (Niaounakis and Halvadakis, 2006; Borja et al.2006)

Considerando la naturaleza, complejidad y variabilidad del alpechín, se han publicado muchos trabajos referidos a diferentes métodos de tratamiento y eliminación (Hachicha, et al.2009; Kapellakis, 2006). En realidad, el verdadero problema es enfrentarse a los costos requeridos para la implementación de esos tratamientos; autores como Hachicha et al (2009) y Hytiris, et al., (2004), mencionan como una práctica efectiva y de bajo costo la disposición en pozas de evaporación, con la desventaja de generar impacto visual, malos olores y proliferación de insectos en áreas circundantes; y en caso de precipitaciones abundantes debe considerarse la posibilidad de infiltración y desborde. Esto no es relevante en nuestra región donde el régimen de lluvias es muy bajo, con vientos secos del sector NE y altas temperaturas lo que favorece a una evaporación rápida, impidiendo así, los encharcamientos e infiltraciones. Otra alternativa de bajo costo y viable para la eliminación y depuración del alpechín es utilizar el suelo como un medio para su tratamiento, el cual se basa en la interacción física, química y microbiológica entre los componentes del efluente y los microorganismos del suelo Los efectos del alpechín sobre las características biológicas y físico-químicas del suelo fueron muy bien documentadas por (Mekki et al., 2006; Rinaldi et al., 2003) y). Un aspecto a tener en cuenta es que el suelo debe ser permeable, poroso y que las napas de la zona afectada sean profundas.

Otra posibilidad de aprovechamiento del alpechín es su uso como fertilizante orgánico en suelos agrícolas (Khoufi, et al., 2009; Paredes et al., 2001;). Sin embargo, la adición incontrolada de alpechín sin tratar causa cambios significativos en la estructura del suelo, la actividad microbiana y limita la viabilidad de su uso para agricultura. Sin embargo, la irrigación de campos con alpechín es una técnica económica de disposición que podría ser implementada por pequeñas industrias con sistema de extracción de tres fases; por cierto con riego limitado de aproximadamente $10 \mathrm{~m}^{3} / 1000$ $\mathrm{m}^{2}$ por año (Komilis et al., 2005)

La planta oleícola, en la cual se ha desarrollado el trabajo, utiliza un sistema de extracción continuo de tres fases y no cuenta con una adecuada gestión de los residuos, el orujo se tira a campo o bien se quema y; el alpechín se dispone sin tratamiento previo en una poza de evaporación (50 $\mathrm{m}^{2}$ aproximadamente). La poza no contiene ningún material aislante y presenta una pendiente aproximada de 2 -3 m; esto permite que se acumule un volumen importante de efluente en el centro. Debido a las condiciones climáticas favorables el alpechín experimenta una evaporación natural y al cabo de un tiempo forma un lodo seco. Asimismo, una vez evaporada la fracción líquida queda en la parte superior de la poza, una fracción de lodo de poco espesor entre 10 y $50 \mathrm{~cm}$; esta fracción de la poza se utiliza para evaluar la posible interrelación entre el suelo y el lodo. 
Los efectos fitotóxicos de un material orgánico no estabilizado, pueden deberse a ciertos factores como contenido de amonio, de ácidos orgánicos volátiles, de metales pesados y de sales, que en concentraciones elevadas pueden generar efectos negativos en el desarrollo de plantas, inhibir la germinación de semillas o el crecimiento de raíces (Varnero et al., 2007). Esto ha incentivado el uso de bioensayos con semillas sensibles a fitotóxicos para determinan el índice de germinación (IG), relacionando el porcentaje relativo de germinación y el crecimiento relativo de raíces (Emino y Warman, 2004). Esto permite establecer distintos niveles de fitotoxicidad, severa, moderada y baja o nula (Varnero et al., 2007). Con el fin de estimar la posible variación en el contenido de compuestos fitotóxicos presentes en el alpechín una vez seco, se realizó un ensayo piloto de germinación con semillas de rabanito (Raphanus sativus).

El objetivo de este trtabajo es determinar la composición química del alpechín, que se dispone en una poza de evaporación y evaluar las modificaciones de sus características físico-químicas a través de la interacción física, química y microbiológica entre el suelo y el efluente. La finalidad de este estudio es obtener datos que permitan proponer algún método factible para la eliminación y tratamiento del efluente y su posibilidad de uso agrícola; como fertilizante del olivar.

\section{MATERIALES Y MÉTODOS}

El alpechín objeto de estudio procede de un emprendimiento oleícola ubicado en el Valle Central a unos $50 \mathrm{Km}$ de San Fernando del Valle de Catamarca; y es vertido, a través de una tubería, en una poza de evaporación ubicada aproximadamente a un kilómetro de las instalaciones; se vierte únicamente durante el proceso de producción entre los meses de abril- mayo. Con la finalidad de evaluar las características del alpechín vertido, durante el proceso de producción, se toman muestras a la salida de los decanters ( $A_{0}$ : puro). Asimismo, se analiza el suelo de la zona, como suelo testigo $\left(S_{0}\right)$ y para comparar la influencia del alpechín, que en la etapa de mayor producción alcanza niveles superiores de la poza, es que se analizan las propiedades del suelo cercano al área de influencia del efluente, $\left(2 \mathrm{~m}\right.$ del nivel superior de la poza) $\mathrm{S}_{1}$.

El alpechín vertido se seca y forma un lodo oscuro y para evaluar los posibles cambios en sus propiedades, se toman muestras de éste en el área superior de la poza, entre 1,5 y 2,5 m del nivel de la zona. Estas muestras se realizaron en diferentes puntos de la poza, y a una profundidad de 0 - 20 $\mathrm{cm}$ de la capa externa, ya que es la parte en la cual interactúan el efluente y el lodo con el suelo. El muestreo del lodo fue estacional con una frecuencia aproximada de tres meses $A_{1}$ (10 días), $A_{2}$ (100 días) $\mathrm{A}_{3}$ (190 días) $\mathrm{A}_{4}$ (280 días). El muestreo del suelo y del lodo fue por cuarteo, se mezclaron, secaron al aire y las de suelo se tamizaron a través de una malla $2 \mathrm{~mm}$. Para los análisis se tomaron las muestras por triplicado (cada muestra se dividió nuevamente en tres). Los parámetros físicoquímicos analizados en suelo, lodo y alpechín fueron: $\mathrm{pH}$ en extracto acuoso 1:2,5 con Peachímetro (HORIBA D-21), conductividad eléctrica (CE) en extracto de saturación con un Termoconductímetro (ALTRONIX Mod CTX - 1); sodio ( $\mathrm{Na}$ ) y potasio (K) (Digestión Húmeda con ácido perclórico y nítrico) por Fotometría - Fotómetro de llama ZF 240 ZELTEC.

En la fracción orgánica se analizó materia orgánica (MO): calcinación, por diferencia total de cenizas en Mufla Computarizada INDEF y carbono orgánico (CO): Método de Walkley-Black, (1932), consiste en oxidar el carbono orgánico con exceso de dicromato de potasio y el exceso se valora con sal de Fe (II) por volumetría. El nitrógeno (N) se analizó por Micro Kjeldahl por digestión ácida en Destilador Kjeldahl M RICHTER 1980 (Page et al., 1982) y el fósforo (P) por el método de Olsen, (Olsen et al., 1954) por colorimetría a $\lambda=882 \mathrm{~nm}$ en un Espectrofotómetro UV- METROLAB 330, usando como extractante bicarbonato sódico a pH: 8,2. Para estimar la materia orgánica oxidable en el alpechín se analizó la evolución de la demanda química de oxígeno (DQO): mediante el Método de oxidación de dicromato de potasio, se utilizó un reactor COD y un colorímetro $\mathrm{HACH}$. Asimismo, la cantidad de oxígeno necesaria para biodegradar la materia orgánica presente en el alpechín se evalúa mediante la demanda bioquímica de oxígeno (DBO): por método instrumental de dilución HACH. BOD Track.

Los compuestos fenólicos solubles $(\mathrm{Ph})$ : se determinaron preparando un extracto con agua desionizada en una proporción 1: $25(\mathrm{~m} / \mathrm{v})$ y se agitó por 12 horas. El extracto concentrado se 
cuantificó según el método colorimétrico de Folin Ciocalteau usando como estándar al ácido cafeico (Box, 1983) y se leyó a una $\lambda=765$ nm en un Espectrofotómetro UV- METROLAB 330.

Para estimar la fitotoxicidad transcurrido 280 día de secado, se realizaron ensayos de germinación de semillas de rabanitos (Raphanus sativus) con etanol al $50 \%$ (Zucconi et al., 1981). Los ensayos se realizaron en siembra directa en distintos sustratos: 1 ( $75 \% \mathrm{~A}_{1}-25$ suelo), 2 ( $75 \% \mathrm{~A}_{2}-25$ suelo), 3 $\left(75 \% \mathrm{~A}_{3}-25 \%\right.$ suelo), $4\left(75 \% \mathrm{~A}_{4}-25 \%\right.$ suelo) y en extractos acuosos de cada muestra. Para las siembras se utilizaron como soporte placas de Petri de $10 \mathrm{~cm}$ de diámetro, en las cuales se colocaron las mezclas sólidas secas $(1,2,3,4)$ y se humedecieron al $60 \%$ dejando reposar 30 minutos; y se sembraron 8 semillas en cada una. Luego se preparó un extracto acuoso con cada muestra $(1,2,3,4)$ añadiendo $13,5 \mathrm{~mL}$ de agua desionizada por gramo de muestra seca para diluir hasta el $10 \%$ y se filtró $(0,45 \mu \mathrm{m}$ poro). En placas cubiertas con papel de filtro se sembraron 8 semillas y se añadió $1 \mathrm{~mL}$ de cada extracto; se realizaron 10 repeticiones incubando a $27^{\circ} \mathrm{C}$ por 48 hs. en oscuridad. Luego, para detener el crecimiento de la planta se agregó $1 \mathrm{~mL}$ de etanol/agua (1 $\mathrm{v} / \mathrm{v}$ ) y se cuantifica el $\mathrm{N}^{0}$ de semillas germinadas $(\mathrm{G})$ y la longitud (L) alcanzada por las raíces por placa. Los resultados se expresan como índice de germinación (IG) el cual se obtiene a partir de a ecuación (1)

IG $(\%)=(\% \mathrm{G} \times \% \mathrm{~L}) \times 100$

Los análisis estadísticos de los datos se determinaron mediante la aplicación del Programa estadístico SPSS, versión 11.0 para Windows. Se aplica la prueba de análisis de varianza paramétrica, como no se cumple el supuesto de homogeneidad de varianza; se aplica entonces análisis de Kruskal - Wallis, pertenecientes al análisis de varianza no paramétrica.

\section{RESULTADOS Y DISCUSIÓN}

Los resultados de los parámetros determinados para las muestras de suelo $\left(\mathrm{S}_{0}\right.$ : testigo $)$ y $\left(\mathrm{S}_{1}: 2 \mathrm{~m}\right.$ de la poza) se muestran la tabla 1.

Tabla 1: Características de las muestras de suelo (\% m.s.)

\begin{tabular}{|l|l|l|l|l|l|l|l|l|}
\hline & \multicolumn{9}{|c|}{ Parámetros } \\
\hline Muestras & $\mathrm{pH}$ & $\mathrm{CE}(\mathrm{dS} / \mathrm{m})$ & $\mathrm{Na} \mathrm{g} / \mathrm{k}_{0}$ & $\mathrm{~K} \mathrm{~g} / \mathrm{k}_{0}$ & $\mathrm{~N} / \mathrm{k}_{0}$ & $\mathrm{P} \mathrm{g} / \mathrm{k}_{0}$ & $\% \mathrm{MO}$ & $\mathrm{CO} \mathrm{g} / \mathrm{k}_{0}$ \\
\hline $\mathrm{S}_{0}$ (Testigo) & 6,65 & 1,43 & 0,35 & 0,40 & 0,04 & 2,19 & 1,70 & 9,9 \\
\hline $\mathrm{S}_{1}(2$ m de la poza) & 6,12 & 1,92 & 0,32 & 0,72 & 0,05 & 1,72 & 4,00 & 11,5 \\
\hline
\end{tabular}

Teniendo en cuenta el valor de la conductividad y la relación de absorción de sodio determinada (RAS, $S_{0}: 0,74$ y $S_{1}: 0,78$ ) se lo puede clasificar como un suelo no salino y no sódico; con un pH que tiende a la neutralidad. La determinación de la textura se realizó al tacto, clasificándolo como suelo Franco Arenoso. Desde el punto de vista nutricional se lo puede clasificar como un suelo pobre ya que presenta valores bajos de carbono orgánico y nitrógeno; mientras que el contenido de fósforo es adecuado. La variación de los valores en los parámetros de la muestra $S_{1}$ indica un ligero descenso del $\mathrm{pH}$, aumento en la concentración de potasio y carbono orgánico, se puede inferir que el alpechín impacta sobre las características del suelo, en la época de mayor producción y mayor generación del efluente, que en ocasiones desborda en el área cercana a la poza (1-2 m). La composición del alpechín analizado se muestra en la tabla 2. Correlacionando los valores de los parámetros analizados se puede decir que el efluente puede clasificarse como levemente ácido con un valor de $\mathrm{pH}: 4,86$ un poco más bajo que para Khoufi et al., (2009) y Alburquerque et al., (2004) con valores 5,32 y 5,20 respectivamente. La carga orgánica evaluada en términos de DQO es algo menor a los obtenidos por Martínez-García et al., (2007), cuya DQO es 90,0 g/L, del mismo modo el poder fitotóxico del alpechín evaluado por la concentración de polifenoles resulta menor a los de los alpeorujos utilizados en Khoufi et al., (2009) y Hachicha et al., (2009) $11,5 \mathrm{~g} / \mathrm{L}$ y $10,5 \mathrm{~g} / \mathrm{L}$ respectivamente. En la tabla 3 se muestran las modificaciones físico-químicas que experimenta el efluente con respecto al tiempo de secado. 
Tabla 2: Características del efluente (salida del decánter)

\begin{tabular}{|l|l|l|l|l|l|l|l|l|l|l|l|}
\hline & \multicolumn{10}{|c|}{ Parámetros } \\
\hline Muestra & $\mathrm{pH}$ & $\mathrm{CE} \mathrm{dS} / \mathrm{m}$ & $\mathrm{Na} \mathrm{g} / \mathrm{k}_{\mathrm{g}}$ & $\mathrm{Kg} / \mathrm{k}_{\mathrm{g}}$ & $\mathrm{Ng} / \mathrm{k}_{0}$ & $\mathrm{P} \mathrm{g} / \mathrm{k}_{\mathrm{g}}$ & $\% \mathrm{MO}$ & $\mathrm{CO} \mathrm{g} / \mathrm{k}_{\mathrm{g}}$ & $\mathrm{Ph} \mathrm{g} / \mathrm{k}_{\mathrm{g}}$ & $\mathrm{DQOg} / \mathrm{L}$ & $\mathrm{DBOg} / \mathrm{L}$ \\
\hline Alpechín & 4,86 & 9,24 & 1,40 & 20,8 & 2,03 & 0,77 & 91,43 & 56,80 & 9,920 & 81,60 & 50,50 \\
\hline
\end{tabular}

Tabla 3: Modificación de los parámetros físico-químicos del alpechín a lo largo del tiempo

\begin{tabular}{|l|l|l|l|l|l|}
\hline & \multicolumn{5}{|c|}{ Muestras } \\
\hline Parámetros & $\mathrm{A}_{0}$ (Decanter) & $\mathrm{A}_{1}$ (10 días) & $\mathrm{A}_{2}$ (100 días) & $\mathrm{A}_{3}$ (190 días) & $\mathrm{A}_{4}$ (280 días) \\
\hline $\mathrm{pH}$ & $4,86 \pm 0,01$ & $5,45 \pm 0,05$ & $6,12 \pm 0,03$ & $6,37 \pm 0,15$ & $6,75 \pm 0,12$ \\
\hline $\mathrm{CE}(\mathrm{dS} / \mathrm{m})$ & $9,24 \pm 0,04$ & $5,20 \pm 0,03$ & $3,06 \pm 0,04$ & $2,47 \pm 0,07$ & $1,96 \pm 0,06$ \\
\hline $\mathrm{N} \mathrm{g} / \mathrm{k}_{0}$ & $2,03 \pm 0,11$ & $0,22 \pm 0,02$ & $0,14 \pm 0,03$ & $0,10 \pm 0,02$ & $0,05 \pm 0,01$ \\
\hline $\mathrm{P} \mathrm{g} / \mathrm{k}_{0}$ & $0,77 \pm 0,01$ & $0,34 \pm 0,03$ & $0,21 \pm 0,04$ & $0,21 \pm 0,04$ & $0,12 \pm 0,01$ \\
\hline $\mathrm{Na} \mathrm{g} / \mathrm{k}_{0}$ & $1,40 \pm 0,01$ & $1,30 \pm 0,03$ & $0,90 \pm 0,00$ & $0,70 \pm 0,00$ & $0,50 \pm 0,01$ \\
\hline $\mathrm{K} \mathrm{g} / \mathrm{k}_{0}$ & $20,8 \pm 0,08$ & $19,7 \pm 0,07$ & $16,7 \pm 0,04$ & $12,0 \pm 0,29$ & $9,10 \pm 0,02$ \\
\hline
\end{tabular}

En la tabla 3; se aprecia que, a excepción del pH, todos los parámetros evaluados disminuyen con el tiempo. El aumento del $\mathrm{pH}$ podría relacionarse con la hidrólisis de sales alcalinas como el sodio y el potasio mencionado por Paredes et al., (2001), esto explicaría la disminución de la concentración del sodio y del potasio. Asimismo, se podría inferir que el aumento del pH es consecuencia de la degradación de ácidos orgánicos como lo ácidos carboxílicos y ácidos fenólicos como se expone en Hachicha et al., (2009). La CE es alta respecto a la salinidad que requieren las plantas para su crecimiento, sin embargo, es bajo respecto de Hachicha, et al (2009) (CE: 20,10 dS/m) y SierraLlopart et al., (2000) (CE 16,24 dS/m), pero es menor al expuesto en Komilis et al., (2005) dS/m: 8,26 esto se corresponde a las características variables del efluente. De todos modos se muestra en la tabla 3 una notable disminución en los valores de CE con el tiempo; Huang et al. (2004) explica que la volatilización de amonio y la precipitación de sales minerales podrían ser las causas de esta disminución.

El efluente es pobre en $\mathrm{N}$ llegando a valores bajos al cabo de los 280 días, con una pérdida de aproximadamente el $97 \%$, esto puede atribuirse a diferentes procesos biológicos y a la volatilización de amoníaco. El descenso de la concentración de fósforo puede deberse a una posible infiltración a perfiles más profundo como lo expresa Sierra et al., (2001) o por inmovilidad, debido a que; a pH cercanos a 6 es posible la formación de fosfato de calcio insoluble. Por no verificar los supuestos del ANOVA paramétrico se aplicó Prueba de Kruskal-Wallis obteniéndose $X^{2}=35$ y $p<0,055$, se puede establecer que las diferencias de los rangos medios de las muestras del efluente $A_{0}$ y los lodos $A_{3} y$ $\mathrm{A}_{4}$ son estadísticamente significativas. La variación de los parámetros determinados para el análisis de la fracción orgánica del efluente y lodo con el tiempo figuran en la tabla 4.

Tabla 4: Fracción orgánica del alpechín analizado a lo largo del tiempo

\begin{tabular}{|l|l|l|l|l|l|}
\hline & \multicolumn{5}{|c|}{ Muestras } \\
\hline Parámetros & $\mathrm{A}_{0}$ (Decanter) & $\mathrm{A}_{1}(10$ días $)$ & $\mathrm{A}_{2}(100$ días $)$ & $\mathrm{A}_{3}$ (190 días) & $\mathrm{A}_{4}(280$ días) \\
\hline CO g / $\mathrm{k}_{0}$ & $56,8 \pm 0,15$ & $52,9 \pm 0,15$ & $51,10 \pm 0,17$ & $38,03 \pm 0,07$ & $33,30 \pm 0,27$ \\
\hline $\mathrm{MO} \%$ & $91,43 \pm 0,61$ & $87,30 \pm 0,63$ & $26,79 \pm 0,58$ & $7,67 \pm 0,20$ & $6,13 \pm 0,08$ \\
\hline $\begin{array}{l}\mathrm{Ph} \mathrm{g} / \mathrm{k}_{0} \\
\text { (polifenoles) }\end{array}$ & $9,920 \pm 4,16$ & $8,300 \pm 2,52$ & $6,100 \pm 3,21$ & $2,100 \pm 1,00$ & $1,200 \pm 1,76$ \\
\hline DBO (g/L) & $50,50 \pm 0,51$ & $32,10 \pm 0,32$ & $19,70 \pm 0,80$ & $11,05 \pm 0,06$ & $2,96 \pm 0,10$ \\
\hline DQO (g/L) & $81,60 \pm 0,82$ & $52,30 \pm 0,52$ & $35,20 \pm 1,43$ & $19,47 \pm 0,10$ & $3,45 \pm 0,12$ \\
\hline
\end{tabular}


La MO experimenta una disminución importante a partir de los 100 días, este comportamiento puede atribuirse a la intensa actividad de los microorganismos, los cuales degradan la fracción de carbono soluble (grasas, carbohidratos, polifenoles solubles y compuestos orgánicos fácilmente biodegradados no identificados) como lo expresa Hachicha et al., (2009). Aún así, al cabo de 280 días mantiene concentraciones interesantes de $\mathrm{MO}$ y $\mathrm{CO}$; ; lo cual resulta favorable a la hora de utilizar el residuo como fertilizante del olivar. Por último, se observa que a los 280 días aún queda una fracción de polifenoles solubles 1,21\%; $\left(\mathrm{A}_{4}\right)$ que junto a la fracción lipídica se relacionan con la fitotoxicidad y el efecto antimicrobiano del alpechín (Alburquerque et al., 2004).

Los índices de germinación, para siembra en el sustrato sólido (mezclas: 1, 2, 3, 4) han mostrado que las muestras 1 y 2 resultaron fitotóxicas debido a que las tazas de germinación fueron nulas en 1 $(0 \%)$ y muy bajas en la muestra $2(10 \%)$; mientras que los sustratos $3(60 \%)$ y $4(80 \%)$ muestran una baja o nula fitotóxicidad ya que las tazas de germinación fueron mayores, en especial en el sustrato 4 cuyos parámetros son similares al suelo original. Para esta clasificación se sigue el criterio de Zucconi et al. (1981), quien considera que un IG $\geq 80 \%$ indica ausencia de compuestos fititóxicos o están en muy baja concentración; si IG $\leq 50 \%$, indica que hay abundancia de sustancias fitotóxicas y si el valor está entre $50 \%$ y $80 \%$ se considera una cantidad moderada de estas sustancias. A la prueba de toxicidad no se aplicó ningún tratamiento estadístico ya que se realizó a modo de prueba piloto.

\section{CONCLUSIONES}

De los resultados obtenidos en este trabajo se puede concluir que en condiciones anaeróbicas el suelo ejerce cierto poder depurador sobre el efluente, lo que se refleja en la disminución de la CE, del $\mathrm{pH}$ que alcanzan valores cercanos a los del suelo de la zona. Asimismo, los índices de germinación después de un período de biodegradación resultan aceptables en las muestras $A_{3}(190)$ y $A_{4}(280$ días), lo que indica una clara disminución de la fitotoxicidad, con una reducción importante en la concentración de polifenoles, los cuales permanecen sólo en un 1,21\%.

Si se analizan las características del efluente puro no se recomienda su uso como fertilizante sin previo tratamiento. Sin embargo, considerando las altas concentraciones de potasio y materia orgánica resultan beneficiosas para el mejoramiento de los suelos, ya que la mayoría de los olivares se encuentran implantados en zonas de suelos pobres en materia orgánica y por otro lado el olivo requiere suelos ricos en potasio.

Teniendo en cuenta que los análisis se realizaron en la zona de la poza con poco espesor de lodo, donde la acción del suelo es factible, se recomienda que las pozas sean poco profundas, y de dimensiones pequeñas que favorecido por las condiciones climáticas con régimen bajos de lluvias y vientos secos se evapore en menos tiempo, e interaccione con el suelo formando una mezcla que sea útil para los olivares.

\section{REFERENCIAS}

Alburquerquer, J. A., y otros tres autores, Agrochemical Characterisation of "Alpeorujo", a Solid ByProduct of the Two-Phase Centrifugation Method for Olive Oil Extraction, Bioresource Technology: 91, 195-200 (2004)

Borja, R.;y otros 2 autores, Treatment Technologies of Liquid and Solid Waste from Two-phase Olive oil mills, Grasas y Aceites: 5(1), 32-46 (2006)

Box,J. D, Investigation of the Folin-Ciocalteau Phenol Reagent for the Determination of Ppolyphenolic substances in natural waters. Water Resources: 17 (5). 511-525. (1983)

Emino, E., P Warman, Biological assay for Compost Quality, Compost Science \& Utilization: 12 (4) 342-348 (2004). 
Hachicha, $\mathrm{S}$ y otros seis autores, Elimination of Polyphenols Toxicity from Olive Mill Wastewater Sludge by its Co-composting with Sesame bark, Journal of Hazardous Materials: 161 (2-3) 1131-1139 (2009)

Huang, G. F.y otros tres autores, Effect of C/N on Composting of Pig Manure with Sawdust, Waste Manage: 24 805-813 (2004).

Hytiris, N.,y otros tres autores, The Potencial use of Olive Mill Sludge in Solidifation Process, Res. Conserv. Recycl : 40 ,129-139 (2004)

Kapellakis, K.P.y otros tres autores, Olive Mill Wastewater Management in River Basins: a Case Study in Greece, Agric. Water Manage: 82, 354-370 (2006).

Khoufi, S., Aloui, F. y Sayadi, S., Pilot Scale Hybrid Process for Olive Mill WasteWater Ttreatment and Reuse, Chemical Engineering and Processing: Process Intensification: 48 (2) 643-650 (2009)

Komilis, D.P.y otros dos autores, The Effect of Olive Mill Wastewater on Seed Germination alter Varioust Pretreatment Techniques. Journal of Enviromental Management: 74 339-348 (2005)

Martínez-García, G. y otros cuatro autores, Olive Waste as a Biosorbent for Heavy Metals, International Biodeterioration \& Biodegradation: 58 (3-4), 331-238 (2006)

Martínez-García, G. y otros cinco autores, Two-Stage Biological Treatment of Olive Mill Wastewater with Whey as Co-Substrat , International Biodeterioration \& Biodegradation: 59 (4), 279-282 (2007)

Mekki, A., A. Dhouib and S. Sayadi, Changes in Microbial and Soil Properties following Amendment with Treated and Untreated Olive mill wastewater,Microbiol. Res.:161 (2) 93-101 (2006).

Niaounakis, M. and Halvadakis, C. P: Olive Processing Waste Mangement, $2^{\text {a }}$ edición, 23-60, ELSEVIER Lta., London UK (2006)

Niaounakis, M. and Halvadakis, C: P. Olive- Mill Waste Mangement Literature review and Patent Survey.Typothito - George Dardanos Publications, Athens, Greece (2004)

Olsen, S.R. y otros tres autores, Estimation of Availabe Phospurus in Soil by Extraction with Sodium Bicarbonate, Department of Agriculture. Circular 938 U.S. Whashington, D.C. (1954)

Page, A. L. y otros dos autores, Methods of Soil Analysis: Chemical and Microbiological Proper ties, American Society of Agronomy. Inc. Madison. Wiskonsin. USA 595 - 624 (1982).

Paredes, M.P., y otros tres autores, Effect of Olive Mill Wastewater Addition in Composting of Agoindustrial and Urban Waste, Biodegradation: 12, 225-234 (2001)

Rinaldi, M., G.,Rana and M. Introna, Olive-mill Wastewater Spreading in Southern Italy: Effects on Adurum Wheat Crop, Field Crops Res.: 84,319-326 (2003)

Sierra Llopart, J. y otros cinco autor, Aprovechamiento del Alpechín a través del Suelo, Estimación del Posible Impacto sobre las Aguas de Infiltración, Edafología: 7 (2), 91-101 (2000)

Sierra,J. y otros cuatro autores, M.A, Characterisation and evolution of a soil affected by olive oil mill wastewater disposal, The Science of the Total Environment: 279 (1-3), 207-214 (2001)

Varnero, M.T., C., Rojas, R., Orellana., Indices de Fitotoxicidad en Residuos Orgánicos durante el Compostaje, R.C. Suelo Nutr. Veg.: 7, 28-37 (2007).

WalKey, A y I.A., Black, An Examination of Degtjareff Method for Determining Soil Organic Matter an Proposed Modifications of the Chromic Acid Titration Method, Soil Science:37 29-38 (1932).

Zucconi,F.,M.Forte, A. Monaco, M. De Bertoldi, Evaluating Toxicity of in Nature Compost, Biocycle: 22, 54-57 (1981) 